\title{
Improved Muller approximate solution of the pull-off of a sphere from a viscoelastic substrate
}

\author{
M. Ciavarella \\ Politecnico di BARI. DMMM department. Viale Gentile 182, 70126 Bari. \\ Mciava@poliba.it
}

\begin{abstract}
The detachment of a sphere from a viscoelastic substrate is clearly a fundamental problem. In the case viscoelastic dissipation is concentrated at the contact edge, and the work of adhesion follows a quite popular simplified model, Muller has suggested an approximate solution, which however is based on an empirical observation. We revisit Muller's solution and show it leads to very poor fitting of the actual full numerical results, particularly for the radius of contact at pull-off, and we suggest an improved fitting of the pull-off which works extremely well over a very wide range of withdrawing speeds, and correctly converges to the JKR value at very low speeds.
\end{abstract}

Keywords:

Viscoelasticity, Adhesion, JKR theory, soft matter

\section{Introduction}

The problem of viscoelastic dissipation during crack growth or contact peeling has attracted much interest due to its fundamental importance in many areas of science and technology. Many authors have applied fracture mechanics concepts and made extensive measurements (Gent and Schultz, 1972, Barquins and Maugis 1981, Gent, 1996, Gent \& Petrich 1969, Andrews \& Kinloch, 1974, Barber et al, 1989, Greenwood \& Johnson, 1981, Maugis \& Barquins, 1980, Persson \& Brener, 2005) postulating peeling involves an effective work of adhesion $w$ as the product of adiabatic value $w_{0}$ and a function of velocity of peeling of the contact/crack line and temperature, namely

$$
w=w_{0}\left[1+k\left(a_{T} v_{p}\right)^{n}\right]
$$

Preprint submitted to journal

December 14, 2020 
where $k, n$ are constants of the material, with $n$ in the range $0.1-0.8$ and $a_{T}$ is the WLF factor (Williams, Landel \& Ferry, 1955) which permits to translate results at various temperatures $T$ from measurement at a certain standard temperature. The details of the derivation from crack models involving cohesive Barenblatt zones or models "truncating" or "blunting" crack tip dissipation (Barber Donley and Langer 1989, Greenwood and Johnson, 1981, Persson \& Brener, 2005) vary, but the form (1) remains the most popular simple choice, and therefore a baseline for comprehension of possible mechanics of contact and crack problems.

In the case of adhesive contact of the fundamental spherical geometry, various authors (Barquins \& Maugis, 1981, Greenwood \& Johnson, 1981, Muller, 1999) have attempted to apply the fracture mechanics formulation with the model (1), and some approximate results have been given in terms of explicit dependences of the pull off force or work, contact radius and approach at pull-off see ref. (Muller, 1999), which we shall revisit here in comparison with full numerical simulation, finding very significant discrepancies, and suggesting some improved fitting of the numerical results, at least for the pull-off force which is the quantity of greater interest.

\section{Spherical contact mechanics theory}

The fracture mechanics formulation for the adhesive contact problem for a sphere is classic, and we shall revisit here only the essentials. We consider the

stress intensity factor at the contact edge is due to the difference between $P_{1}$, the load required to maintain a contact radius $a$ in the absence of adhesion

$$
P_{1}(a)=\frac{4}{3} \frac{E^{*}}{R} a^{3}
$$

where $E^{*}=E /\left(1-\nu^{2}\right)$ is the plane strain elastic modulus ( $E$ being Young's modulus and $\nu$ Poisson's ratio) and $P$, the smaller load to maintain the same contact radius in the presence of adhesion. So we find the strain energy release rate as 1

$$
G(a, P)=\frac{K(a, P)^{2}}{2 E^{*}}=\frac{\left(P_{1}(a)-P\right)^{2}}{8 \pi E^{*} a^{3}}
$$

\footnotetext{
${ }^{1}$ The factor 2 which is missing in Muller (1999) comes from the fact that strain energy exists only in one material, assuming the other is rigid. For two identical materials, $\frac{1}{E^{*}}=\frac{2}{E_{1}^{*}}$ and we return to the standard LEFM case with $G(a)=\frac{K(a)^{2}}{E_{1}^{*}}$.
} 
In the adhesionless conditions, the remote approach is $\alpha_{1}(a)=\frac{a^{2}}{R}$, so in the adhesive condition we have to decrease this by an amount given by a flat punch displacement $\Delta \alpha=\frac{P_{1}-P}{2 E^{*} a}$ (since in moving from the adhesionless to the adhesive solution we keep the contact area constant) giving the general result for approach

$$
\alpha(a, P)=\frac{a^{2}}{R}-\frac{P_{1}(a)-P}{2 E^{*} a}
$$

from which we can obtain $P(a, \alpha)$ using (2)

$$
\begin{aligned}
P(a, \alpha) & =P_{1}(a)+2 E^{*} a \alpha(a, P)-2 E^{*} \frac{a^{3}}{R} \\
& =\frac{2 E^{*} a}{R}\left(R \alpha(a, P)-\frac{a^{2}}{3}\right)
\end{aligned}
$$

which corresponds to Muller (1999) equation 10, whereas using (3)

$$
G(a)=\frac{\left(P_{1}(a)-P\right)^{2}}{8 \pi E^{*} a^{3}}=\frac{E^{*}}{2 \pi a R^{2}}\left(R \alpha(a)-a^{2}\right)^{2}
$$

which corresponds to Muller (1999) equation 15 except for a factor 2 misprint. For the elastic case, JKR (Johnson, Kendall \& Roberts, 1971) theory is obtained by using (마) and (41)

$$
P=\frac{4}{3} \frac{E^{*}}{R} a^{3}-\sqrt{8 \pi w_{0} E^{*} a^{3}}
$$

Putting

$$
\zeta=\left(\frac{\pi w_{0}}{6 R E^{*}}\right)^{1 / 3}
$$

we have at $P=0$ from (17) and (15)

$$
\begin{aligned}
a_{0} & =\left(\frac{9}{2} \pi R^{2} \frac{w_{0}}{E^{*}}\right)^{1 / 3}=3 R \zeta \\
\alpha_{0} & =\frac{a_{0}^{2}}{3 R}=3 R \zeta^{2}
\end{aligned}
$$

where there is a factor 3 misprint in Muller (1999) equation 19. 


\section{Viscoelasticity}

Now, for a viscoelastic material, the material dissipation at the crack tip/contact edge requires that energy balance imposes the velocity of crack according to (1). Further, we can write the velocity of the contact edge as

$$
v_{p}=-\frac{d a}{d t}=v \frac{d a}{d \alpha}
$$

where $v$ is the remote pull-off rate imposed by the loading equipment. The condition $G(a)=w$ therefore defines a differential equation for $a=a(\alpha)$ obtained using (6, 11)

$$
\frac{1}{k^{1 / n} a_{T} v}\left[\frac{E^{*}}{2 \pi a R^{2} w_{0}}\left(R \alpha(a)-a^{2}\right)^{2}-1\right]^{1 / n}=\frac{d a}{d \alpha}
$$

By using we the JKR values at zero load (9210) and the JKR values for pull-off for $P_{0}=\frac{3}{2} \pi R w_{0}$, and finally the adiabatic work of adhesion for $G$, we obtain the dimensionless variables

$$
G^{\prime}=\frac{G}{w_{0}} ; \quad P^{\prime}=\frac{P}{P_{0}} ; \quad a^{\prime}=\frac{a}{a_{0}} ; \quad \alpha^{\prime}=\frac{\alpha}{\alpha_{0}}
$$

If we now remove the (') for simplicity in the following equation s, we rewrite (12) as

$$
\frac{d a}{d \alpha}=\beta^{-1}\left[a^{3}\left(\frac{\alpha}{3 a^{2}}-1\right)^{2}-\frac{4}{9}\right]^{1 / n}
$$

where we have introduced the only dimensionless factor in the problem, apart from $n$, namely

$$
\beta=\left(\frac{6 R E^{*}}{\pi w_{0}}\right)^{1 / 3}\left(\frac{4 k}{9}\right)^{1 / n} a_{T} v
$$

The latter two equation s correspond to Muller (1999) equation 24,23. The differential equation (14) can be solved for initial conditions starting from a point on the loading curve2, which is the JKR curve which in this dimensionless notation and in parametric form is

$$
P(a)=4\left(a^{3}-a^{3 / 2}\right)
$$

\footnotetext{
${ }^{2}$ Strictly speaking, during loading adhesion is reduced with respect to the adiabatic value at zero speed, but we neglect this effect, or else we consider that loading occurs near thermodynamic equilibrium.
} 
and

$$
\alpha(a)=3 a^{2}-2 a^{1 / 2}
$$

After $a(\alpha)$ is obtained, we can compute the load which in dimensionless form is obtained from

$$
P(a, \alpha)=2 a\left(\alpha-a^{2}\right)
$$

Notice that the strain energy release rate in dimensionless form is

$$
G=\frac{9}{4} a^{3}\left(\frac{\alpha}{3 a^{2}}-1\right)^{2}
$$

\subsection{Muller's approximate solution}

Muller (1999) in searching for the pull-off as the minimum of the $P(\alpha)$ curve, postulates that this is close to the minimum of $P(\alpha)+G(\alpha)$ which is also 0 in the minimum. There is no fundamental reason for this mix of the dimensionless load with the dimensionless strain energy release rate to have any special property, and indeed we found the two minima are not necessarily very close. Muller's postulate anyway leads to radius of contact, approach and load at pull-off,

$$
\begin{aligned}
& a_{m}=\kappa \beta^{q} \\
& \alpha_{m}=-\kappa^{2} \beta^{2 q} \\
& P_{m}=\left|P_{\min }\right|=4 \kappa^{3} \beta^{3 q}
\end{aligned}
$$

where $q=n /(n+3)$ and $\kappa=\left(\frac{9 / 16}{4^{n}}\right)^{1 /(n+3)}$. Notice obviously that this result at zero velocity would give incorrect results as all values go to zero, rather than the asymptotic values of JKR theory for thermodynamic equilibrium.

Remark that the actual velocity of the crack line (recall $a$ and $\alpha$ are dimensionless here, not to be confused with equation 11)

$$
\frac{v_{p}}{v}=\frac{1}{\zeta}\left(\frac{d a}{d \alpha}\right)_{m}=\frac{1}{\zeta} \frac{1}{4 a_{m}}
$$

and given $a_{m} \sim 1$ while $\zeta<<1$, it is clear that $\frac{v_{p}}{v}>>1$ so that the velocity at the contact line can be much greater than the cross-head remote velocity, which permits to make the approximation that the bulk may be essentially in a relaxed elastic state. Notice however that, in concentrating the effect of 
dissipation at the crack tip, despite the dissipation can occur very far from it, there is another possible approximation: indeed, the form of solution we are using is unlikely to be reliable at extremely high speeds anyway, also for thermal effects and other possible physical factors.

\section{Numerical results and fittings}

Here we report some results of the numerical solution of the differential equation, comparison with Muller's approximate solution, and some improved fitting results for the pull-off, which is (perhaps) the most important quantity.

From Fig.1 we see the withdrawing curves for an example case of low $n=0.25$, and (b) an example showing that initial conditions seem to very weakly affect the actual pull-off, as Muller had remarked. From Fig.2 we see that the contact radius at pull-off is very poorly predicted by Muller's approximate solution (20), and it is much more weakly dependent on $\beta$. In particular, at high $\beta$, Muller's solution predicts very large $a_{m}$ which do not make much sense. Indeed, as we have seen there is not much dependence on the initial condition, we expect $a_{m}<1$ as when we are unloading from equilibrium condition at zero load, and since we expect the radius to further decrease, a fortiori we obviously end up with a smaller radius that at zero load, which is $a_{i}=1$. An exception, where we see $a_{m}>1$ but not by a large factor, is when there is some weak dependence on initial conditions and we start from very high loads (see example of $P_{i}=5$ of fig.2a,c). At low $\beta$, Muller's prediction underestimates the radius at pull-off, particularly at high $\beta$.

Also not very good predictions, but perhaps better than for contact radius, are those for the approach at pull-off (fig.3). Here, the actual results tend to be higher than Muller's prediction (21), at all speeds, and start off with a value near $\alpha_{m}=-0.5$ rather than from 0 . 

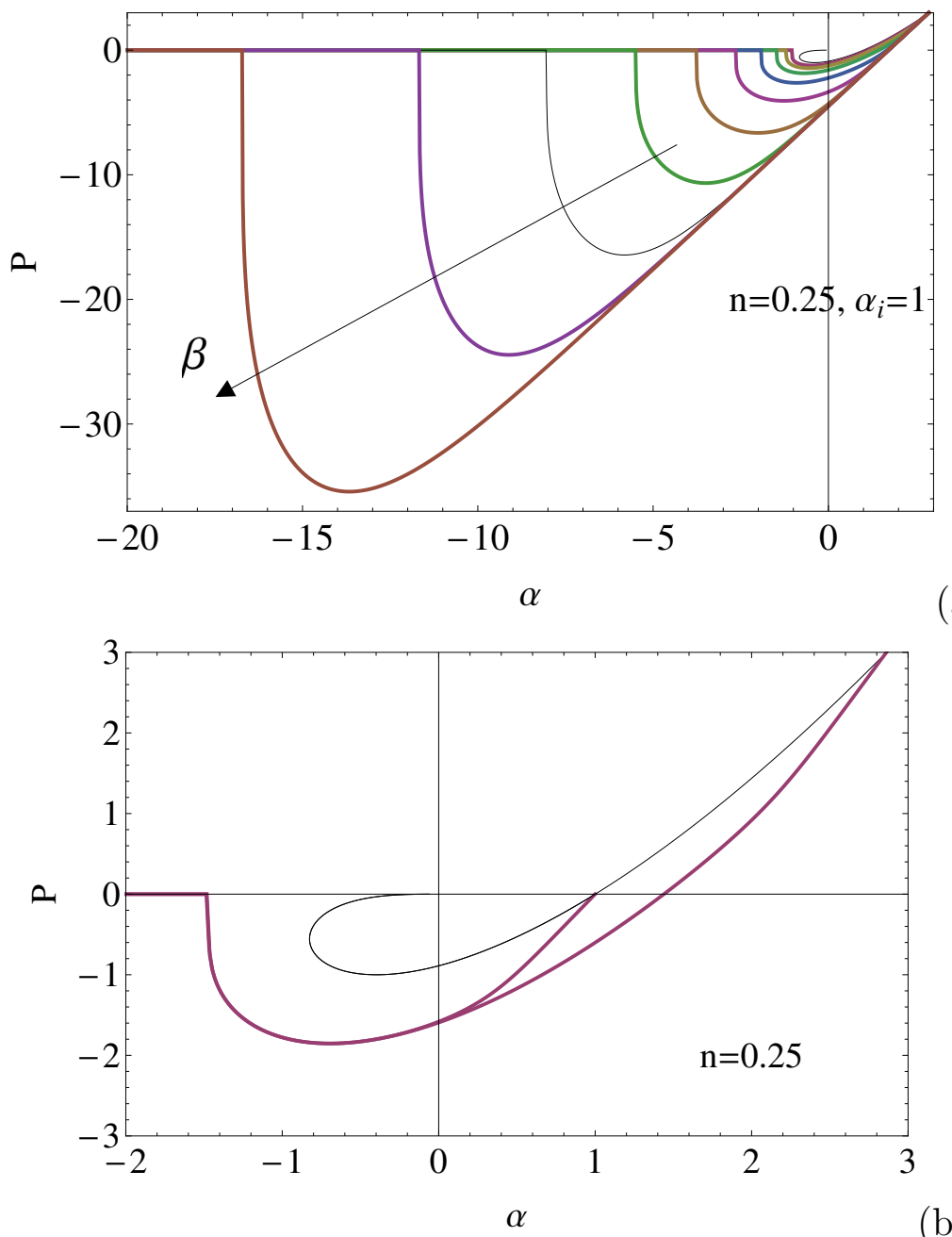

(a)

(b)

Fig.1 - Dimensionless load $P$ dimensionless approach $\alpha$ (a) for various $\beta=2 \times 10^{-5} * 15^{i},(i=1,10)$ and for $n=0.25$. The inner black curve is the adiabatic JKR curve. (b) very weak dependence of pull-off on initial conditions (initial load $P=0,5$ ) for an example case $\beta=0.0675$ 

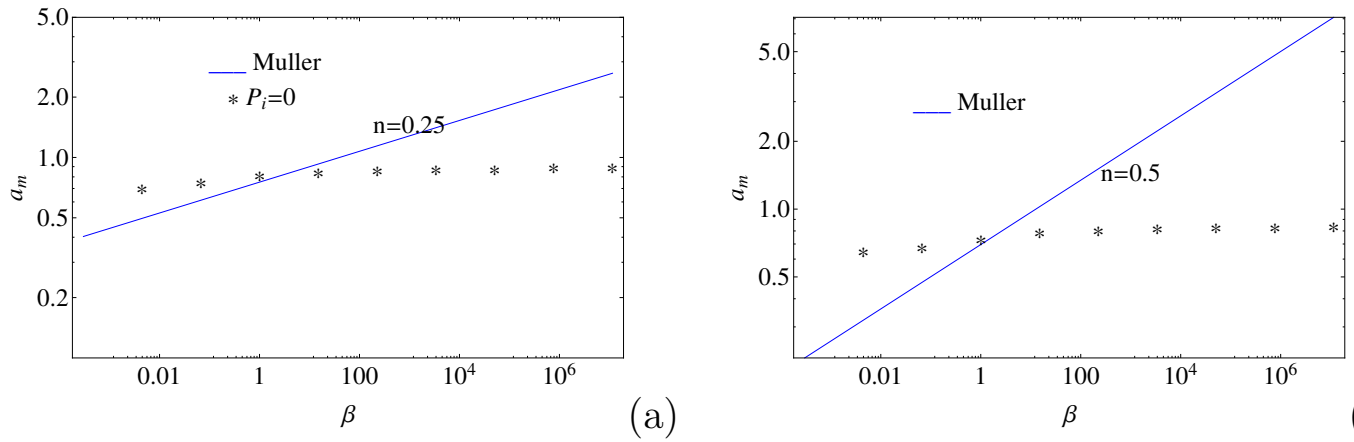

(b)

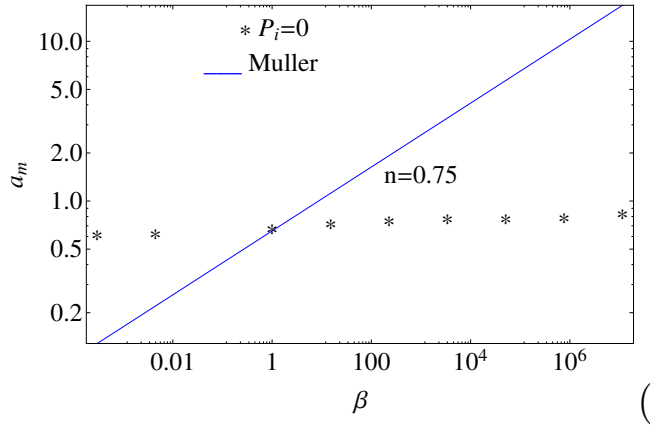

Fig.2 - Dimensionless contact radius at pull-off $a_{m}$ for $n=0.25$ (a) $n=0.5$ (b), $n=0.75$ (c) as a function of the dimensionless speed factor $\beta$. (initial load in the figure $P=0$ or 5 )
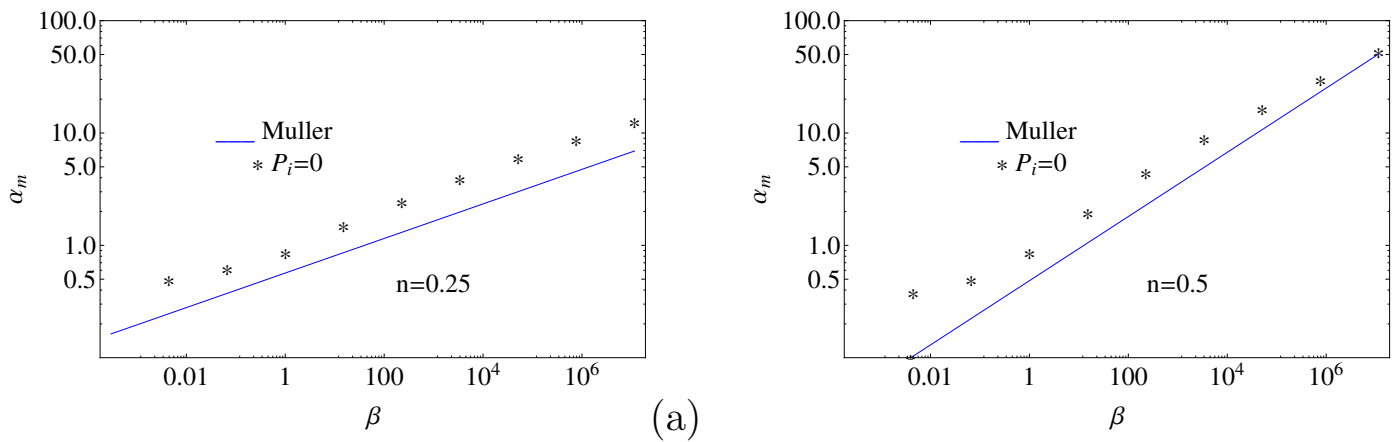

(b) 


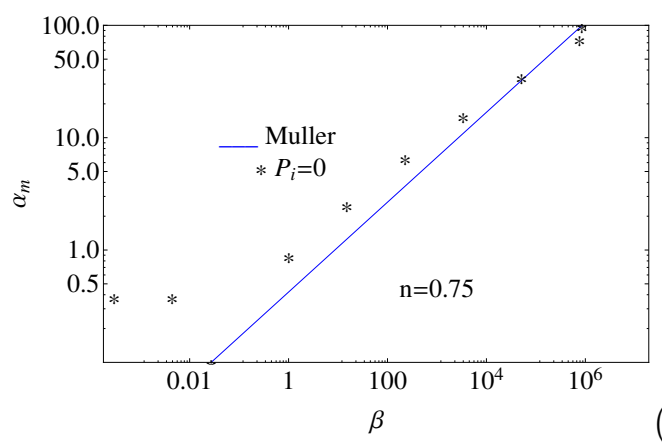

Fig.3 - Dimensionless absolute value of approach at pull-off $\left|\alpha_{m}\right|$ for $n=0.25$ (a) $n=0.5$ (b), $n=0.75$ (c) as a function of the dimensionless speed factor $\beta$. (initial load in the figure $P=0$ or 5 )

Considering these poor performances on $a_{m}$ and $\alpha_{m}$, the results for the pull-off load vs Muller's prediction (see Fig.4) are relatively good (blue line vs the markers of the numerical simulations), which is probably why he was satisfied in his paragraph "comparison with exact calculation" where he has only comparison with pull-off load or work for pull-off, but still we find them only rough "estimates". It is easy to obtain much better fit of the results, considering we have only two independent dimensionless parameters, $n$ and $\beta$ of course, so we improve Muller's prediction in two respects:

1) we add a crossover towards the JKR value $P=1$, by adding " 1 " to Muller's equation (22) the JKR load;

2 ) we improve the power law exponent at large $\beta$ with a corrective factor to Muller's equation (22) in the form

$$
P_{m}=\left|P_{\min }\right|=1+4 \kappa^{3} \beta^{3 q / c(n)}
$$

where

$$
c(n)=1.1+n / 1.65
$$

This improvement shows clearly a much better fit with respect to detailed numerical calculations in the entire range of realistic values for $n$ and of $\beta$ covering 10 orders of magnitude in $\beta$ which is probably more than enough considering the other approximations made in the model, namely the form of the work of adhesion, that there is no viscoelasticity in the bulk, no thermal effects, and so on.

Notice that Violano and Afferrante (2019) have numerically solved the Muller equation s, and found good correlation with experimental results. 
This suggests that our solution would be very valuable for an analytical fitting of experiments such as those of Violano \& Afferrante (2019).
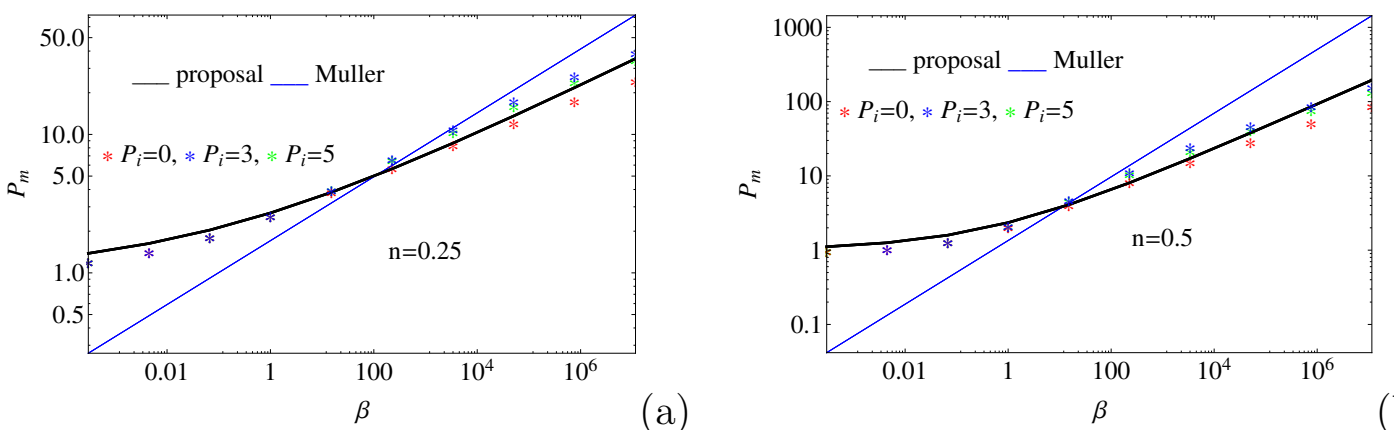

(b)

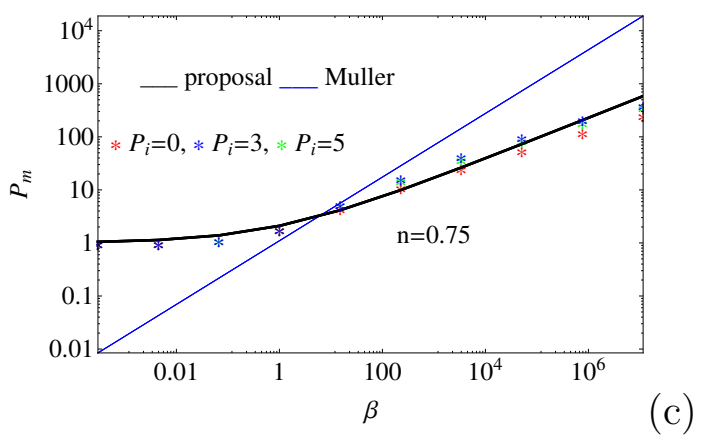

Fig.4 - Absolute value of the dimensionless load at pull off $P_{m}$ for $n=0.25$

(a) $n=0.5$ (b), $n=0.75$ (c) as a function of the dimensionless speed factor

$\beta$. (initial load as indicated by different colors in the markers in the figure $\left.P_{i}=0,3,5\right)$. Blue power law curve is the Muller (1999) prediction (22), while the thick black solid line is our proposal (24).

\section{Conclusions}

We have revisited the Muller approximate solution for the pull-off of sphere from a flat viscoelastic material, finding significant errors in the approximate solution, which stem from the rather arbitrary assumption that the pull-off condition occurs when the sum of a dimensionless load and a dimensionless strain energy release rate has a minimum. We have added a "cross-over" towards the JKR solution for very low velocities, and corrected 
the power law enhancement of pull-off with velocity of withdrawal. The solution can be useful for quick estimates of the effect of viscoelasticity on the increase of adhesion in spherical geometries.

\section{Acknowledgements}

MC acknowledges support from the Italian Ministry of Education, University and Research (MIUR) under the program "Departments of Excellence" (L.232/2016).

\section{References}

Andrews, E. H., \& Kinloch, A. J. (1974). Mechanics of elastomeric adhesion. In Journal of Polymer Science: Polymer Symposia (Vol. 46, No. 1, pp. 1-14). New York: Wiley Subscription Services, Inc., A Wiley Company.

Barber, M., Donley, J., \& Langer, J. S. (1989). Steady-state propagation of a crack in a viscoelastic strip. Physical Review A, 40(1), 366.

Barquins, M., \& Maugis, D. (1981). Tackiness of elastomers. The Journal of Adhesion, 13(1), 53-65.).

Dahlquist, C. A. in Treatise on Adhesion and Adhesives, R. L. Patrick (ed.), Dekker, New York, (1969a), 2, 219

Dahlquist, C., Tack, in Adhesion Fundamentals and Practice. (1969b), Gordon and Breach: New York. p. 143-151.

Gent, A. N., \& Schultz, J. (1972). Effect of wetting liquids on the strength of adhesion of viscoelastic material. The Journal of Adhesion, 3(4), 281-294.

Gent, A. N., \& Petrich, R. P. (1969). Adhesion of viscoelastic materials to rigid substrates. Proceedings of the Royal Society of London. A. Mathematical and Physical Sciences, 310(1502), 433-448.

Greenwood, J. A., \& Johnson, K. L. (1981). The mechanics of adhesion of viscoelastic solids. Philosophical Magazine A, 43(3), 697-711.

Johnson, K.L. , Kendall, K. , Roberts, A.D., (1971) Surface energy and the contact of elastic solids. Proc $R$ Soc Lond : A324:301-313. doi: 10.1098/rspa.1971.0141

Maugis, D., \& Barquins, M. Fracture mechanics and adherence of viscoelastic solids. In: Adhesion and adsorption of polymers. Springer, Boston, MA, 1980. p. 203-277. 
Muller, V. M. (1999). On the theory of pull-off of a viscoelastic sphere from a flat surface. Journal of Adhesion Science and Technology, 13(9), 9991016

Persson, B. N. J., \& Brener, E. A. (2005). Crack propagation in viscoelastic solids. Physical Review E, 71(3), 036123.

Roberts, A. D. (1979). Looking at rubber adhesion. Rubber Chemistry and Technology, 52(1), 23-42.

Violano, G., \& Afferrante, L. (2019). Adhesion of compliant spheres: an experimental investigation. Procedia Structural Integrity, 24, 251-258.

Williams, M. L.; Landel, R. F.; Ferry, J. D. The Temperature Dependence of Relaxation Mechanisms in Amorphous Polymers and Other Glass-Forming Liquids. Journal of the American Chemical Society 1955, 77, 3701-3707. 